\title{
IN-SITU SPECTROSCOPY AND TWO-COLOR THERMOGRAPHY DURING MICROWAVE IRRADIATION IN MATERIALS PROCESSING
}

\author{
J. Fukushima ${ }^{1}$ and H. Takizawa ${ }^{1}$,
}

${ }^{1}$ Graduated school of Engineering, Dept. of Appl. Chem., Tohoku University, 6-6-07 Aoba Aramaki, Sendai, Miyagi 980-8579, Japan

fukushima@aim.che.tohoku.ac.jp

Keywords: microwave selective heating, plasma exciting, in-situ measurement, high spatial resolution

\begin{abstract}
In $\gamma-\mathrm{Fe}_{2} \mathrm{O}_{3}+\mathrm{C}$ system, a plasma discharge (such as $\mathrm{CN}$ and $\mathrm{N}_{2}$ ) during microwave E-field irradiation and local temperature distribution is are investigated by using in situ emission spectroscopy device and high-resolution two-color thermography. The time development of excited plasma in E-field irradiation demonstrate that excited $\mathrm{N}_{2}, \mathrm{C}$ and $\mathrm{CN}$ were observed at first but $\mathrm{CN}$ excitation was sustained at least 3 seconds. In microwave $\mathrm{H}$-field irradiation, $\gamma-\mathrm{Fe}_{2} \mathrm{O}_{3}$ powders were selectively heated compared to carbon. Excited $\mathrm{CN}$ results in enhancement of the reduction reaction in addition to thermal reduction in E-field irradiation, and selective heating of $\gamma-\mathrm{Fe}_{2} \mathrm{O}_{3}$ suggests a reduction reaction proceeds in thermally nonequilibrium condition in $\mathrm{H}$-field irradiation.
\end{abstract}

\section{Introduction}

Microwave processing has many advantageous such as rapid heating and selective heating, resulting it is expected to apply for high-temperature processing including material synthesis $^{1-3}$ and sintering ${ }^{4,5}$. Concentration of microwave E-field between material particles is considered to cause the enhancement of sintering ${ }^{6}$ and chemical reaction under microwave irradiation. For example, it is usually required $1700{ }^{\circ} \mathrm{C}$ to synthesize AIN by carbothermal reduction method using $\mathrm{Al}_{2} \mathrm{O}_{3}$ as a starting material, but microwave processing can proceed this process at $1200{ }^{\circ} \mathrm{C}^{7}$. In particular, microwave smelting has an attracted attention because of the great merit of microwave processing. For example, microwave process can produce a pig iron about $1350{ }^{\circ} \mathrm{C}^{8}$. The process temperature is very low to smelt the phosphorus oxides: the produced slag was decreased in microwave process. Moreover, the reduction of copper oxide $(\mathrm{CuO})$ occurred during microwave irradiation, and the reduction behavior was different between irradiation by the microwave electric field and the magnetic field ${ }^{9}$. In addition, our previous work investigated the effect of microwave E- and H-field on the reduction reaction by in situ visible image and emission spectra during microwave irradiation ${ }^{10}$. These studies suggest that the effects of plasma and microwave electric and magnetic fields itself can contribute to ironmaking reactions, and these effects result in lowtemperature reactions and a shortening of the period of the carbothermal reduction reaction. To understand the mechanism of microwave smelting process, it is necessary to understand an occurrence behavior of plasma, which leads to a chemical reaction related to radical species generated by a local E-field concentration. In addition, in carbothermal reduction reaction, which is typical smelting method, a raw material was composed of several $\mathrm{mm}$ powders. It is suggested that a selective heating in the powder scale occurs. However, to discuss this selective heating behavior on this scale, it is necessary to realize a quantitative temperature measurement system with independent of the emissivity of the material and several mm spatial resolution. 
In this study, we conducted an in-situ spectroscopy and two-color thermography to verify these non-equilibrium effects during microwave irradiation.

\section{Experimental procedure}

In-situ spectroscopic system was explained elsewhere ${ }^{10}$. The authors was used $\gamma-\mathrm{Fe}_{2} \mathrm{O}_{3}$ (99\% purity, 20-40 nm, Kojundo Chemical Laboratory Co., Ltd., Saitama, Japan) and pure carbon (99.9\%, $50 \mu \mathrm{m}$, Kojundo Chemical Laboratory Co., Ltd., Saitama, Japan) as starting powders. The powders were mixed together $\left(\gamma-\mathrm{Fe}_{2} \mathrm{O}_{3}: \mathrm{C}=2: 3\right.$ mol.\%) with alumina mortar and pestle with small amount of ethanol. The mixture powders $(0.15 \mathrm{~g})$ were pressed into a pellet $(\varnothing 6 \mathrm{~mm})$ at a pressure of $4 \mathrm{MPa}$. Microwave irradiation system was composed of magnetron (IMH-20A259, IDX Co., Ltd., Tochigi, Japan, $2455 \pm 15 \mathrm{MHz}$ ), isolator, three stub tuner, TE102 single-mode cavity and plunger. Sample pellet was set in the quartz test tube. During microwave irradiation, pure nitrogen gas was flowed at $0.1 \mathrm{~L} / \mathrm{min}$. Microwave was irradiated with the sample, which is placed at the maximum point of E-filed and H-field intensity in the cavity. The sample temperature of the bottom of the pellet was measured by infrared (IR) thermometer (FTK9-P300A-30L21, Japansensor, Co., Tokyo, Japan).

Visible images and emission spectra were obtained by using an integrated microscopic imaging spectrometer (IMIS, Bunkoukeiki Co., Ltd., Tokyo, Japan) during microwave process ${ }^{11}$. This system can measure the images and spectra on the upper part of the pellet. The spectrometric wavelength ranged from $308 \mathrm{~nm}$ to $492 \mathrm{~nm}$.

In addition, temperature distribution of the sample at upper part of the pellet was obtained by two-color thermography system (Thermera-NIR2, Mitsui photonics Ltd., Tokyo, Japan) during microwave $\mathrm{H}$-field irradiation in five minutes.

Obtained sample was analyzed by X-ray diffraction (XRD) and Field-type emission scanning electron microscopy (FE-SEM) and energy dispersion spectroscopy (EDS). In both analysis, upper surface of the pellet was analyzed; irradiated sample was not pulverized before sample analysis.

\section{Results and discussion}

During E-field irradiation, peripheral edges of the pellet was only heated to a high temperature, and the maximum temperature of the center of the pellet was up to $800^{\circ} \mathrm{C}$, which value was indicated by IR measurement. Microwave power at holding temperature at $800{ }^{\circ} \mathrm{C}$ was about $200 \mathrm{~W}$.

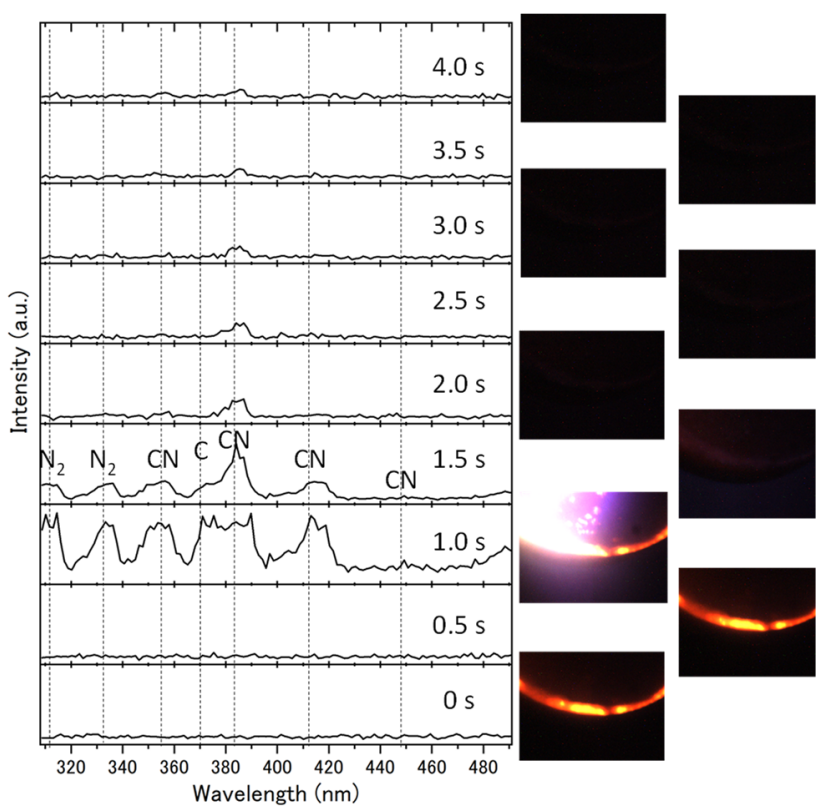

Fig. 1. Visible image of the sample pellet and emission spectra within 4 seconds during microwave E-field processing. 
First of all, we investigated that the time development of plasma when $\gamma-\mathrm{Fe}_{2} \mathrm{O}_{3}+\mathrm{C}$ pellet was irradiated with microwave E-field. Figure 1 shows the visible image of the sample pellet and plasma spectra within 4 seconds. At $0.0 \mathrm{~s}$, peripheral edge was heated up, but no peaks was observed in a spectral pattern: the spectroscopic spectrum at $0.0 \mathrm{~s}$ was almost flat between $308-492 \mathrm{~nm}$. At $1.0 \mathrm{~s}$, white $\sim$ purple plasma was generated as shown in visible image. From the spectra, these peaks were derived from $\mathrm{N}_{2}, \mathrm{CN}$ and $\mathrm{C}$. Then, this plasma seemed to be disappeared from the visible image, but $\mathrm{N}_{2}, \mathrm{CN}$, and $\mathrm{C}$ spectra were remained. On the other hand, hot region on the peripheral edge were almost disappeared, indicating that the temperature at these area decreased. At $2.0 \mathrm{~s}, \mathrm{~N}_{2}$ and $\mathrm{C}$ spectra were almost disappeared, but $\mathrm{CN}$ peaks was remained for 2 minutes. This excited $\mathrm{CN}$ enable to enhance the reduction reaction in addition to thermal reduction.

Figure 2 shows the visible image of the sample pellet and plasma spectra within 4 seconds in microwave $\mathrm{H}$-field irradiation. Unlike E-field process, the temperature of the pellet was increased up to $1200{ }^{\circ} \mathrm{C}$ in $\mathrm{H}$-field irradiation. Moreover, almost all the visible area of the pellet was heated up uniformly, indicating that uniform

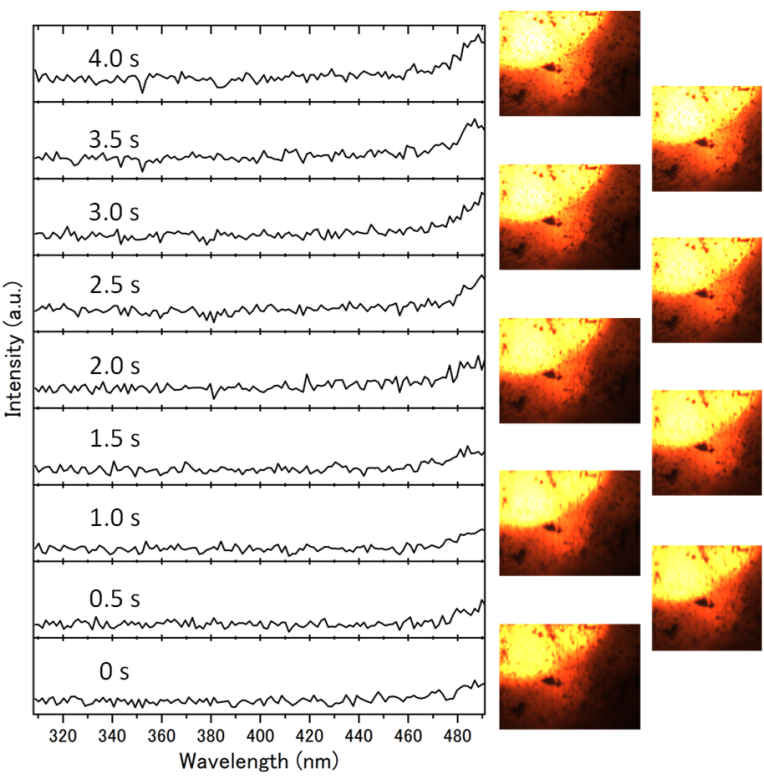

Fig. 2. Visible image of the sample pellet and emission spectra within 4 seconds during microwave $\mathrm{H}$-field processing.

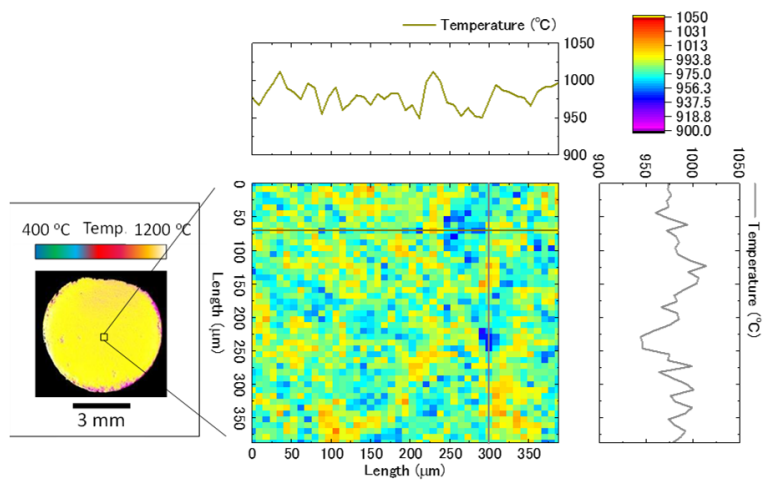

Fig. 3. Two-color thermography at $1000^{\circ} \mathrm{C}$ (measured by IR thermometer) and the local temperature distribution image 
heating was achieved as compared to E-field heating process. The images and spectra was measured at about $1100{ }^{\circ} \mathrm{C}$, and the microwave power at that time was about $400 \mathrm{~W}$. As shown in Fig. 2, continuous Planck radiation was only observed in the high-wavelength range. Furthermore, spectra intensity of these continuous Planck radiation was increased with the IR temperature increasing. During H-field experiment, no peaks was observed; thus, in H-field irradiation, plasma did not occur.

In-situ spectroscopy during microwave process suggest that plasma or excited radicals will affect the reduction reaction when the sample was irradiated with E-field. On the other hand, in H-field, the reduction reaction seems to proceed only thermal reaction.

Then, in order to evaluate the selective heating effect on ironmaking process, the authors focused on in situ measurement of the reduction reaction in $\mathrm{Fe}-\mathrm{O}-\mathrm{C}$ system during microwave irradiation by using two-dimensional two-color thermography. Figure 3 shows that the two-color thermography at $1000{ }^{\circ} \mathrm{C}$ (measured by IR thermometer) and the local temperature distribution image. The two-color thermography image demonstrated that the pellet temperature was almost uniform, but the temperature at peripheral edge of the pellet was little lower than center part of the pellet. The temperature distribution image at about $400 \mu \mathrm{m}$ square part in the pellet was shown in the center of Fig. 3. From the figure, temperature gradient was observed in such a local scale. The temperature profile of the horizontal line indicated in the temperature distribution image was shown at the top of Fig. 3 . The profile suggested that the sample temperature dropped at around $x=275 \mu \mathrm{m}$. The width of this area was corresponds to the size of the pristine carbon powders (about $50 \mu \mathrm{m}$ ). The temperature profile at vertical line was also shown in Fig. 3 right. The temperature was reached a low at around $y=250 \mu \mathrm{m}$. The width of this localized area was also about $50 \mu \mathrm{m}$. These profiles suggested that the carbon powders were existed. In this system, single-phase $\gamma-\mathrm{Fe}_{2} \mathrm{O}_{3}$ and carbon absorbs microwaves strongly. From the above results, in mixture case, $\gamma$ $\mathrm{Fe}_{2} \mathrm{O}_{3}$ is selectively heated in $\mathrm{H}$ field irradiation. Although carbon also absorbs microwaves, a selective heating was occurred in the scale of $\mu \mathrm{m}$ powder.

XRD results of the pristine powders and the sample pellet after microwave irradiation were shown in Fig. 4. The pristine powders certainly consisted of $\gamma-\mathrm{Fe}_{2} \mathrm{O}_{3}$ and carbon, and after microwave process, carbon peaks remained but $\gamma-\mathrm{Fe}_{2} \mathrm{O}_{3}$ became iron and wustite. Therefore, the reduction reaction was not complete in five minutes.

Figure 5 shows FE-SEM image and element mapping by EDS. The sample had many macro pores. Element mapping exhibit that carbon remained; the results is

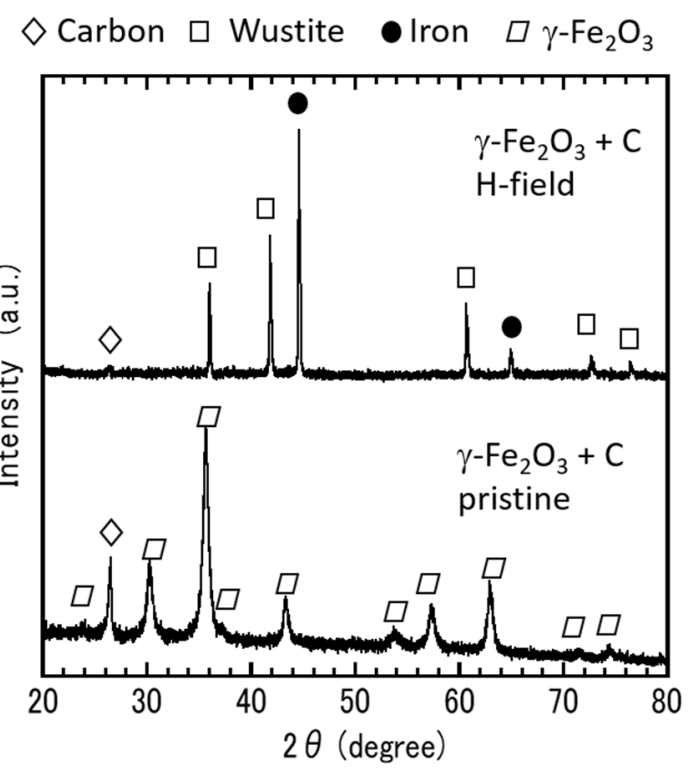

Fig. 4. XRD results of the pristine powders and the sample pellet after microwave irradiation consistent with the XRD results. 
Oxygen mapping demonstrate that the amount of oxygen was low at around remained carbon. This results indicate that the reduction reaction proceeded at the contact point
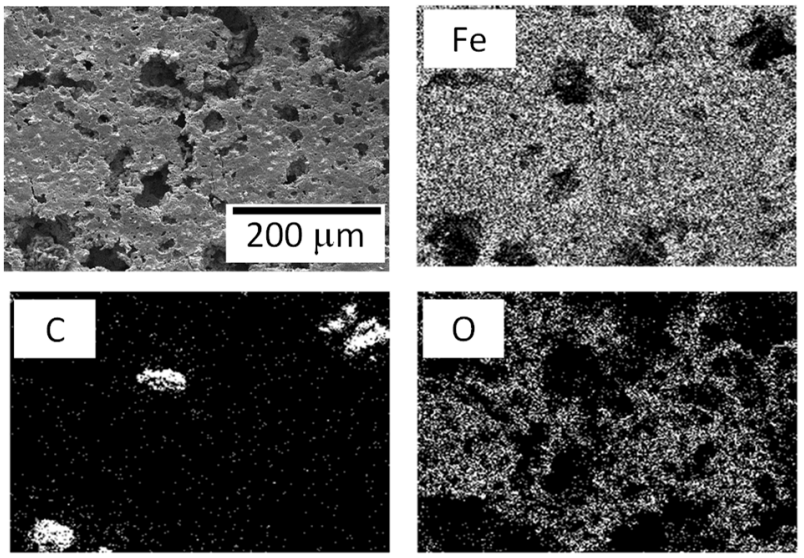

Fig. 5. FE-SEM image and element mapping by EDS between carbon and $\gamma$ $\mathrm{Fe}_{2} \mathrm{O}_{3}$.

Here we discuss the selective heating effect in this system. In $\mathrm{H}$-field heating, $\quad \gamma-\mathrm{Fe}_{2} \mathrm{O}_{3} \quad$ was selectively heated and carbon was heated by thermal conduction from heated $\gamma-\mathrm{Fe}_{2} \mathrm{O}_{3}$. On the other hand, temperature distribution was sustained during microwave $\mathrm{H}$-field irradiation, and the reduction reaction of iron oxides proceeded under thermally non-equilibrium condition.

\section{Conclusion}

The study investigated that the time development of excited plasma in E-field irradiation and selective heating of $\gamma-\mathrm{Fe}_{2} \mathrm{O}_{3}$ in $\mathrm{H}$-field irradiation by using in-situ measurement system which enable to obtain emission spectra and high-resolution two-color thermographic image. In microwave E-field irradiation, excited $\mathrm{N}_{2}, \mathrm{C}$ and $\mathrm{CN}$ were observed, and $\mathrm{CN}$ excitation was sustained at least 3 seconds. This excited $\mathrm{CN}$ causes enhancement of the reduction reaction in addition to thermal reduction. In microwave $\mathrm{H}$-field irradiation, $\gamma-\mathrm{Fe}_{2} \mathrm{O}_{3}$ powders were selectively heated compared to carbon.

\section{Acknowledgement}

This work was supported by a JSPS Grant-in-Aid for Scientific Research (S) No. JP17H06156.

\section{References}

[1] Takeuchi, T. et al., Catalysts, 2017, 7, 65

[2] Kashimura, K. et al., Journal of Alloys and Compounds, 2013, 550 239-244.

[3] Komarneni, S. et al., Mater. Res. Bull., 1992, 27 1393-1405

[4] Sutton, W., Am. Ceram. Soc. Bull., 1989, 68, 376-386.

[5] Roy, R. et al., Nature, 1999, 399, 668

[6] Rybakov, K.I. et al, Abbr. J. Am Ceram. Soc., 2013, 94, 1003-1020.

[7] Chikami, H., Fukushima, J. et al., J. Am Ceram. Soc., 2018, 101, 4905-4910.

[8] Ishizaki, K. et al., ISIJ International, 2006, 46, 1403-1409.

[9] Fukushima J. et al., Materials Chemistry and Physics, 2013, 172, 47-53

[10] Fukushima, J. et al., Metals, 2018, 8, 49-58.

[11] Matsubara, A. et al., Plasma Fusion Res., 2010, 5, 041. 